\title{
Tumefactive Primary Central Nervous System Vasculitis: Imaging Findings of a Rare and Underrecognized Neuroinflammatory Disease
}

\author{
(D) S. Suthiphosuwan, (D)A. Bharatha, (D)C.C.-T. Hsu, (D)A.W. Lin, (D).A. Maloney, (DD.G. Munoz, (D) C.A. Palmer, and (D) A.G. Osborn
}

\begin{abstract}
SUMMARY: Primary central nervous system vasculitis (PCNSV) is a poorly understood neuroinflammatory disease of the CNS affecting the intracranial vasculature. Although PCNSV classically manifests as a multifocal beaded narrowing of the intracranial vessels, some patients may not have angiographic abnormalities. A rare subset of patients with PCNSV present with masslike brain lesions mimicking a neoplasm. In this article, we retrospectively review 10 biopsy-confirmed cases of tumefactive PCNSV (t-PCNSV). All cases of t-PCNSV in our series that underwent CTA or MRA were found to have normal large and medium-sized vessels. TPCNSV had a variable MR imaging appearance with most cases showing cortical/subcortical enhancing masslike lesion (70\%), often with microhemorrhages (80\%). Diffusion restriction was absent in all lesions. In summary, normal vascular imaging does not exclude the diagnosis of t-PCNSV. Advanced imaging techniques including MR perfusion and MR spectroscopy failed to demonstrate specific findings for t-PCNSV but assisted in excluding neoplasm in the differential diagnosis. Biopsy remains mandatory for definitive diagnosis.
\end{abstract}

\footnotetext{
ABBREVIATIONS: PCNSV = primary central nervous system vasculitis; t-PCNSV = tumefactive PCNSV; ABRA = amyloid $\beta$-associated angiitis; CAA-RI = cerebral amyloid angiopathy-related inflammation; $E S R=$ erythrocyte sedimentation rate; MRP $=$ MR perfusion; $C R P=C$-reactive protein; $P C R=$ polymerase chain reaction; $\mathrm{VWI}=$ vessel wall imaging; PCNSL = primary CNS lymphoma
}

$\mathbf{P}$ rimary central nervous system vasculitis (PCNSV) is a poorly understood neuroinflammatory disease involving intracranial vessels. ${ }^{1-5}$ The typical radiologic manifestation of PCNSV is multifocal beading of the large and medium-sized intracranial vessels. An underrecognized and rarer subset of PCNSV, approximately 5\%$29 \%$, can present with "masslike" or "tumefactive" lesions mimicking a neoplasm. 3 ,4,6-25

To date, imaging features of tumefactive PCNSV ( $\mathrm{t}-\mathrm{PCNSV}$ ) have not been well described and definitive diagnosis can only be made confidently on histopathology. ${ }^{5-10}$ In this article, we

Received April 7, 2020; accepted after revision May 26.

From the Departments of Medical Imaging (S.S., A.B., C.C.-T.H., A.W.L.), Surgery (A.B.), and Laboratory Medicine and Pathobiology (D.G.M.), University of Toronto, Toronto, Ontario, Canada; Department of Medical Imaging (C.C.-T.H.), Gold Coast University Hospital, Queensland, Australia; Department of Radiology (J.A.M.), University of Colorado, Aurora, Colorado; and Departments of Pathology (C.A.P.), and Radiology and Imaging Sciences (A.G.O.), University of Utah, Salt Lake City, Utah.

This study was selected as an oral scientific presentation at: 57th Annual Meeting, of the American Society of Neuroradiology, May 2019; Boston, Massachusetts (presentation\# 2550)

Please address correspondence to Suradech Suthiphosuwan, MD, FRCR, Neuroradiologist and Assistant Professor, Department of Medical Imaging, Unity Health-St. Michael's Hospital, University of Toronto, 30 Bond St, Toronto, ON, M5B 1W8, CANADA; e-mail: suradech.suthiphosuwan@unityhealth.to

Indicates article with supplemental on-line table.

http://dx.doi.org/10.3174/ajnr.A6736 performed a retrospective review of 10 histopathologically proved cases of t-PCNSV and analyzed pertinent imaging features with histopathologic correlation.

\section{CASE SERIES}

\section{Case Selection}

We performed a retrospective pathology data base search by using the keywords "brain biopsy" and "vasculitis" in histopathology reports from July 2010-December 2018 at a single institution. Exclusion criteria included patients with histopathology findings of amyloid-beta $(\mathrm{A} \beta)$-associated angiitis (ABRA) or cerebral amyloid angiopathy-related inflammation (CAA-RI), and infectious CNS vasculitis. Finally, we identified 6 patients who had a final diagnosis of t-PCNSV from this institution. The other 4 patients with t-PCNSV were collected from the imaging and histopathology archives of the contributing authors from 2 other institutions.

All 10 patients presented with masslike brain lesion(s) with provisional radiologic diagnoses of brain neoplasm on initial MR imaging studies, and all patients had eventual histopathologic confirmation of t-PCNSV. Patient records were retrospectively reviewed, and demographic features, clinical presentation, histopathology, laboratory results, and followup studies were noted. 


\section{Patient Demographics}

\begin{tabular}{|c|c|}
\hline Age, median; range (years) & $42(16-68)$ \\
\hline Sex, male (\%) & $7(70 \%)$ \\
\hline \multicolumn{2}{|l|}{ Clinical presentations, $n(\%)$} \\
\hline Headache & $4(40 \%)$ \\
\hline Seizure & $3(30 \%)$ \\
\hline Hemiparesis & $3(30 \%)$ \\
\hline Confusion & $2(20 \%)$ \\
\hline Visual disturbance & $2(20 \%)$ \\
\hline Cognitive impairment & $1(10 \%)$ \\
\hline Word-finding difficulty & $1(10 \%)$ \\
\hline \multicolumn{2}{|l|}{ Location of involvement, $n(\%)$} \\
\hline Cortex/subcortical white matter & 7 (70\%) \\
\hline Deep white matter & $6(60 \%)$ \\
\hline Basal ganglia & $4(40 \%)$ \\
\hline Brain stem & $1(10 \%)$ \\
\hline \multicolumn{2}{|l|}{ Enhancement patterns, $n(\%)$} \\
\hline Patchy enhancement & $5(50 \%)$ \\
\hline Small nodular enhancement & $5(50 \%)$ \\
\hline Ring enhancement & $2(20 \%)$ \\
\hline Linear/perivascular enhancement & $1(10 \%)$ \\
\hline Leptomeningeal enhancement & $4(40 \%)$ \\
\hline Subependymal enhancement & $3(30 \%)$ \\
\hline Microhemorrhages, $n(\%)$ & $8(80 \%)$ \\
\hline Patients with available CSF analysis, $n(\%)$ & $5(50 \%)$ \\
\hline Abnormal CSF protein $(>0.5 \mathrm{~g} / \mathrm{L})$ & $3(60 \%)$ \\
\hline CSF leukocytosis ( $>5$ cells $/ \mathrm{mm}^{3}$ ) & $4(80 \%)$ \\
\hline Patients with available serum inflammatory marker, $n(\%)$ & $8(80 \%)$ \\
\hline High ESR $(>20 \mathrm{~mL} / \mathrm{h})$ & $2(25 \%)$ \\
\hline High CRP (>5 mg/L) & $2(25 \%)$ \\
\hline \multicolumn{2}{|l|}{ Surgical procedure } \\
\hline Stereotactic-guided biopsy & $5(50 \%)$ \\
\hline Tumor resection & $4(40 \%)$ \\
\hline Open wedge biopsy & $1(10 \%)$ \\
\hline \multicolumn{2}{|l|}{ Histopathology } \\
\hline Lymphocytic vasculitis & $7(70 \%)$ \\
\hline Lymphocytic plus necrotizing vasculitis & $2(20 \%)$ \\
\hline Granulomatous vasculitis & $1(10 \%)$ \\
\hline \multicolumn{2}{|l|}{ Treatment } \\
\hline Steroid alone & $5(50 \%)$ \\
\hline Steroid plus immunosuppressive agents & $5(50 \%)$ \\
\hline Total duration follow-up ${ }^{\mathrm{a}}$, median (range), months & $18(4-77)^{a}$ \\
\hline
\end{tabular}

${ }^{a}$ Three patients did not have available clinical follow-up detail.

\section{Imaging Analysis}

Two neuroradiologists (S.S. and C.C.-T.H.) reviewed all anonymized CT and MR imaging studies in a consensus analysis. Brain MRIs were performed on either $1.5 \mathrm{~T}$ or $3 \mathrm{~T}$ scanners. In all studies, MR imaging sequences performed included T1-weighted imaging, T2-weighted imaging, FLAIR, and DWI. Depending on the scanner used, susceptibility-based sequences, either $\mathrm{T} 2^{\star}$ weighted gradient-echo imaging (GRE-T2*) or susceptibilityweighted imaging, were performed. Gadolinium-enhanced T1WI was acquired in all patients. Location, configuration, signal characteristics, and enhancement pattern of the lesions were recorded. When available, vascular imaging studies including CTA and MRA (9/10) were assessed. In some patients, advanced MR imaging techniques including MR perfusion (MRP) (3/10), MR spectroscopy (2/10), and vessel wall imaging (VWI) (3/10) findings were available.

\section{RESULTS}

\section{Clinical Demographics}

The 10 patients ranged in age from 16 to 68 years with a mean age of 43.5 years (median, 42 years) at the time of initial diagnosis. There were 7 (70\%) males and 3 (30\%) females.

Eight patients did not have pre-existing medical conditions. One patient had known alcoholic cirrhosis and hypertension. One patient had known hypoglossal nerve injury from a prior thyroglossal duct cyst resection.

Initial clinical presentations of t-PCNSV were: headaches, 4 (40\%); followed by seizures, 3 (30\%); hemiparesis, 3 (30\%); confusion, 2 (20\%); visual disturbance, 2 (20\%); cognitive impairment, 1 (10\%); and word-finding difficulty 1 (10\%).

Five (50\%) patients had available CSF analysis. Of these, 4 (80\%) had CSF leukocytosis (ranging from 7-47 cells $/ \mathrm{mm}^{3}$ ), and 3 (60\%) had abnormal CSF protein (ranging from $0.54-0.71 \mathrm{~g} / \mathrm{L}$ ). Serum inflammatory markers including erythrocyte sedimentation rate (ESR) and C-reactive protein (CRP) were available in 8 (80\%) patients. Two had elevated ESR: 22 and $45 \mathrm{~mm} / \mathrm{h}$. Two had evaluated CRP: 10 and $32 \mathrm{mg} / \mathrm{L}$.

All patients had available systemic autoimmune and infectious laboratory work-up, some of which included: HIV antibody, HSV1-2, HZV polymerase chain reaction (PCR), hepatitis B and C antibodies, tuberculosis PCR, Venereal Disease Research Laboratory test, antinuclear antibodies, anti-neutrophil cytoplasmic antibodies, C3, C4, and rheumatoid factor; these available results were within normal limits.

Patient clinical demographics, laboratory findings, and treatment are summarized in Table 1 and detailed in the On-line Table.

\section{Imaging Findings}

Locations and Multiplicity. Eight (80\%) patients presented with a single brain lesion (Figs 1-3). Two (20\%) patients presented with multifocal lesions. Lesion locations in the brain included: cortex/subcortical white matter in 7 patients (70\%), deep and/or periventricular white matter in 6 patients (60\%) (Figs 1 and 2), basal ganglia in 4 patients (40\%) (Fig 3), and brain stem in 1 patient $(10 \%)$.

Signal Characteristics. All lesions had a hypointense signal on T1WI and intermediate to hyperintense signal on T2WI. Marked perilesional T2WI high signal of vasogenic edema was seen in 5 (50\%) patients. Five $(50 \%)$ patients had moderate perilesional vasogenic edema. Diffusion restriction was absent in all lesions (Fig 3B).

Eight $(80 \%)$ patients had intralesional microhemorrhages, seen as small linear or punctate patterns on either GRE-T2* or SWI (Fig 1E-H). Two (20\%) patients did not have evidence of intralesional hemorrhages. Microhemorrhages were not encountered diffusely elsewhere in the brain.

Enhancement Patterns. All 10 patients (100\%) demonstrated enhancing lesions. Five patients (50\%) demonstrated patchy parenchymal enhancement. Three of them demonstrated "mottled appearance" characterized by multiple small hypoenhancing areas within the patchy enhancing masses (Figs $1 A-C$ ). Small nodular 

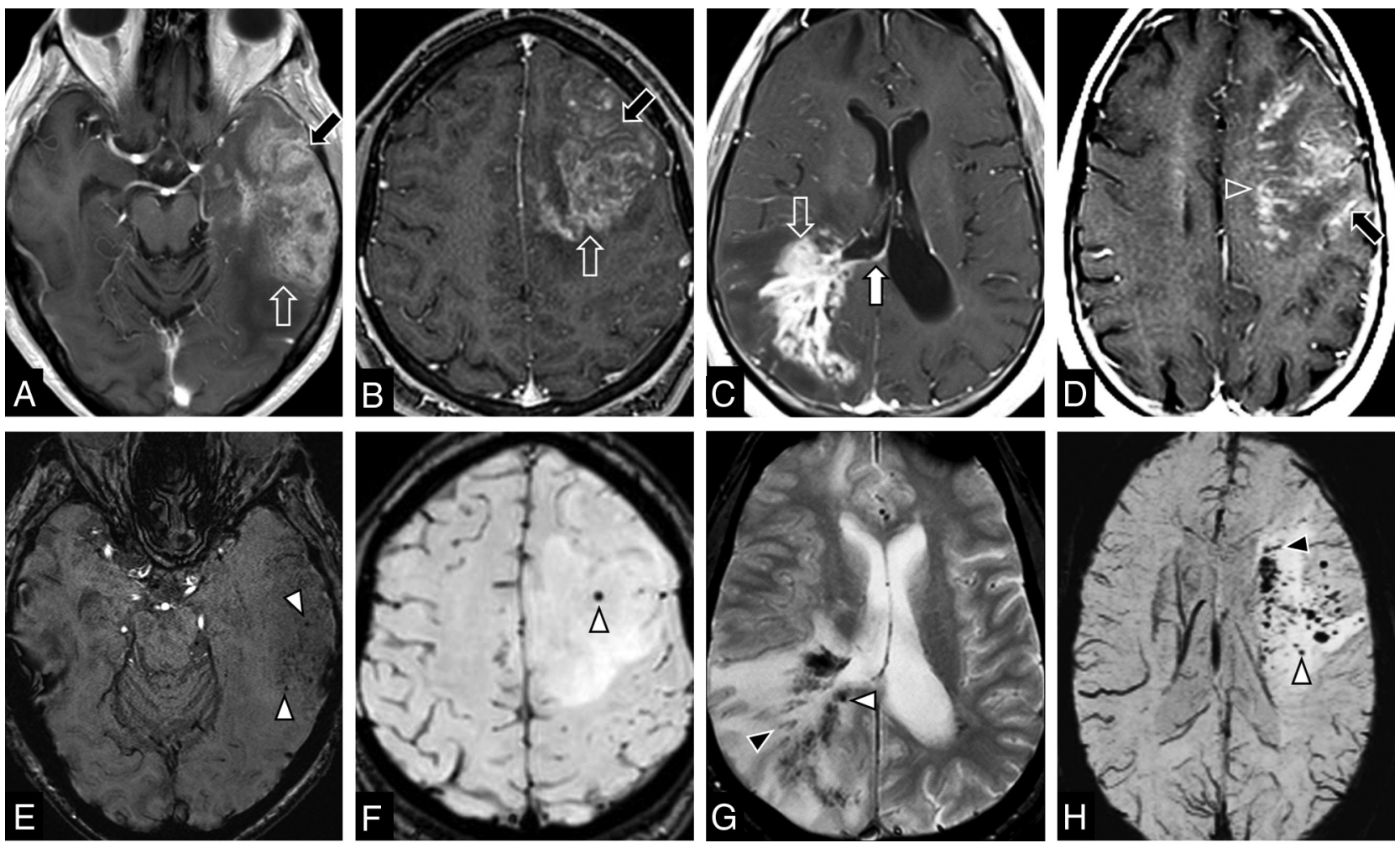

FIG 1. Common MR imaging features of t-PCNSV in 4 different patients. $A$ and E: 68-year-old man (case 5), $B$ and F: 16-year-old male adolescent (case 6), $C$ and G: 42-year-old man (case 8); and $D$ and $H$ : 55-year-old woman (case 7). Axial postcontrast T1-weighted images (A-D) demonstrate lobar masses. The masses have patchy heterogeneous enhancement (mottled appearance) on $A-C$ (open arrow) and nodular and linear (perivascular enhancement) on $D$ (open arrowhead). Leptomeningeal enhancement is noted on $A, B$, and $D$ (black arrows). Subependymal enhancement is observed on $C$ (white arrow). Axial susceptibility-weighted imaging $\left(E, F\right.$, and $H$ ) and axial $T 2^{*}$-weighted gradient-echo imaging $(G)$ demonstrate microhemorrhages. Punctate microhemorrhages are shown in $E-H$ (white arrowheads). Linear microhemorrhages are demonstrated on $G$ and $H$ (black arrowheads).

enhancement was found in 5 patients (50\%) (Figs $1 D$ and $2 B$ ). Two patients (20\%) had ring enhancement (Figs $3 A$ and D). Linear (perivascular) enhancement pattern was found in 1 patient $(10 \%)$ (Fig 1D).

Localized leptomeningeal enhancement adjacent to the dominant/largest lesions was found in 4 patients (40\%) (Figs $1 A, B$, and $D)$. Localized subependymal enhancement adjacent to the lesions was found in 3 patients (30\%) (Figs 1C, $3 A$, and $3 D$ ), who had lesions extended to the ventricular margin. No patient had diffuse leptomeningeal enhancement or diffuse subependymal enhancement.

Vascular Imaging: CTA, MRA, and VWI. Nine (90\%) of 10 patients had available cerebral CTA and/or MRA. All findings (100\%) were normal. None of the patients underwent conventional angiography.

Three patients (30\%) had VWI and the imaged proximal large intracranial arteries had no abnormal wall thickening or enhancement.

Advanced MR Imaging: MRP and MR Spectroscopy. Three patients (30\%) had available MRP; all demonstrated low relative cerebral blood volume (Fig 3E). Two patients (20\%) underwent MR spectroscopy, both demonstrated elevated choline peaks compared with the creatine and slightly decreased NAA peak (choline/ creatine ratios were approximately 2.0, and choline/NAA ratios were approximately 0.9 ). One patient demonstrated a high lipidlactate peak while the other did not have a lipid-lactate peak.

\section{Histopathologic Findings}

All patients underwent surgery: 5 patients (50\%) had a stereotactic-guided biopsy, 4 patients (40\%) underwent gross total resection, and 1 patient (10\%) had an open wedge biopsy.

Histopathologically, t-PCNSV is characterized by transmural inflammation of small blood vessels in the brain parenchyma and/or leptomeninges, inducing parenchymal ischemic changes of varying ages. ${ }^{26}$

In 9 patients (90\%), the lesions were described as lymphocytic vasculitis, which is characterized by a vasculocentric lymphocytic inflammatory infiltrate confirmed on immunohistochemistry staining: CD20 for B-cell lymphocytes (Fig 2E) and CD3 for Tcell lymphocytes (Fig $2 F$ ). Two of the patients with lymphocytic PCNSV had necrotizing vasculitis, characterized by extensive transmural fibrinoid necrosis of the vessel wall confirmed by Martius Scarlet blue staining. ${ }^{26}$

One patient (10\%) had granulomatous t-PCNSV (Fig 3) characterized by vasculocentric predominantly mononuclear cell infiltration accompanied by multinucleated giant cells and fibroblasts. ${ }^{26}$

None of these lesions demonstrated features of amyloid depositions on $\mathrm{H} \& \mathrm{E}$ staining. Confirmatory immunohistochemistry staining for $\mathrm{A} \beta$ and/or Congo red were available in 7 patients 

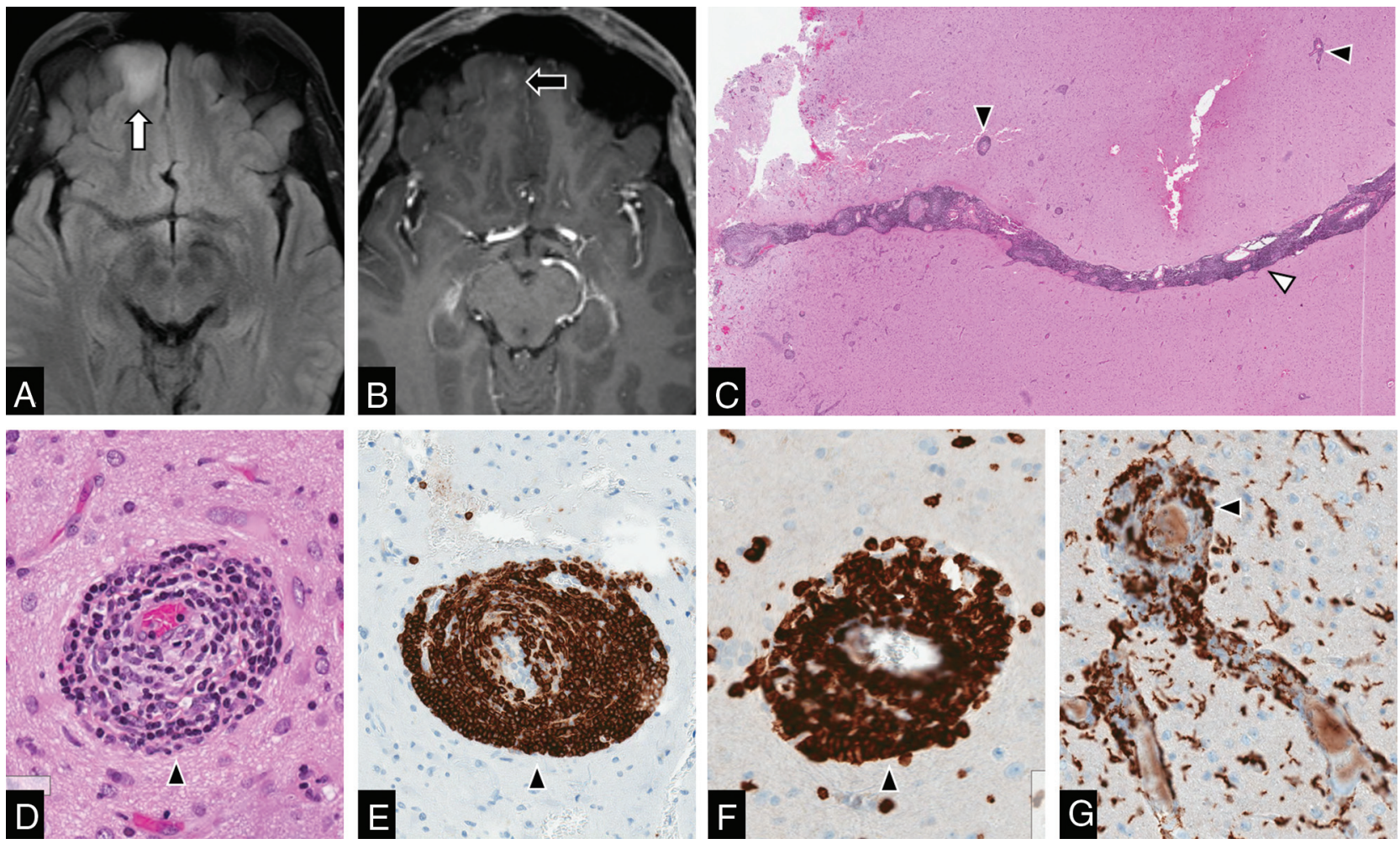

FIG 2. MR imaging findings with histopathologic correlation of t-PCNSV. A 42-year-old man (case 1) presented with first episode seizure. Axial fluid-attenuated inversion recovery $(A)$ shows a hyperintense lesion involving the cortex and subcortical white matter (white arrow). Axial post gadolinium T1-weighted imaging (B) demonstrates that the lesion has small nodular enhancement (black arrow). The initial imaging diagnosis was glioma. This patient underwent a gross total resection of the mass. Hematoxylin and eosin-stained ( $C: 2 \mathrm{~mm}$ and $D: 200 \mu \mathrm{m}$ magnifications) demonstrated vasculocentric transmural lymphocytic infiltration involving the leptomeningeal artery ( $C$, white arrowhead) and parenchymal cortical arteries ( $C$ and $D$, black arrowheads). Immunohistochemically, the most infiltrating lymphocytic cell is positive for CD20 marker for B-cell lymphocytes $(E)$ and CD3 marker for T-cell lymphocytes $(F)$. The minority of inflammatory cells are macrophages that are positive for CD163 (G).

and were negative. None of these lesions showed nuclear atypia or polymorphic cells. Also, all lesions had Epstein-Barr encoding region in situ hybridization for the detection of the Epstein-Barr virus and were negative.

\section{Medical Treatment and Follow-Up}

Five patients received corticosteroid treatment alone and 5 patients had a combination of corticosteroid and immunosuppressive agents that included cyclophosphamide and/or azathioprine. Seven patients had available clinical and imaging followup, with a median total duration of follow-up interval of 18 months (range: 4-77 months). Six patients had complete remission on the latest follow-up. One patient had recurrent disease at 48 months after initial treatment and had a remission after repeat treatment with corticosteroid and immunosuppressive therapy (Fig 3).

\section{DISCUSSION}

Our study describes and illustrates the spectrum of imaging findings of t-PCNSV, which is a rare disorder manifesting as masslike brain lesions. T-PCNSV seems to preferentially involve small parenchymal and leptomeningeal vessels, usually sparing large to medium-sized intracranial vessels, which may appear unremarkable on commonly performed imaging studies such as CTA or MRA.
Radiologic features of t-PCNSV were not well described or recognized because it is extremely rare entity. ${ }^{4}$ To our knowledge, apart from several case reports ${ }^{11-22}$ and review articles, ${ }^{23-25}$ only a few small case series have been published in the medical literature. ${ }^{6-8,10}$ Molloy et $\mathrm{al}^{7}$ reported 8 cases of masslike PCNSV with the proved histopathology including both "A $\beta$-related" $\mathrm{PCNSV}$ and "non-A $\beta$-related" PCNSV from 2 tertiary institutions. In addition, they included a literature review of 30 cases. Lee et $\mathrm{al}^{10}$ reported 4 cases of biopsy-proved lymphocytic PCNSV mimicking brain tumor on MR imaging. de Boysson et $\mathrm{al}^{8}$ described 10 masslike PCNSV cases in their series. Nine cases had histopathologyconfirmed PCNSV; 1 case had histopathology negative for PCNSV but had large and medium vessel abnormalities suggestive of PCNSV on both MRA and DSA. In their series, they did not specify the absence or presence of $\mathrm{A} \beta$ on histopathology. Recently, Salvarani et $\mathrm{al}^{6}$ have reported a comprehensive analysis of $13 \mathrm{bi}$ opsy-proved cases of masslike PCNSV; however, 6 were found to have $\mathrm{A} \beta$-related PCNSV (or ABRA/CAA-RI), leaving only 7 non$\mathrm{A} \beta$-related PCNSV cases. None of these case series identified definitive radiologic features of t-PCNSV. Furthermore, the inclusion of $\mathrm{A} \beta$-related PCNSV or ABRA/CAA-RI in their sample size may preclude their analysis of the radiologic features of non- $\mathrm{A} \beta$-related PCNSV. In contrast, our study is exclusively focused on neuroimaging features of $\mathrm{t}$-PCNSV with histopathologic correlation and 

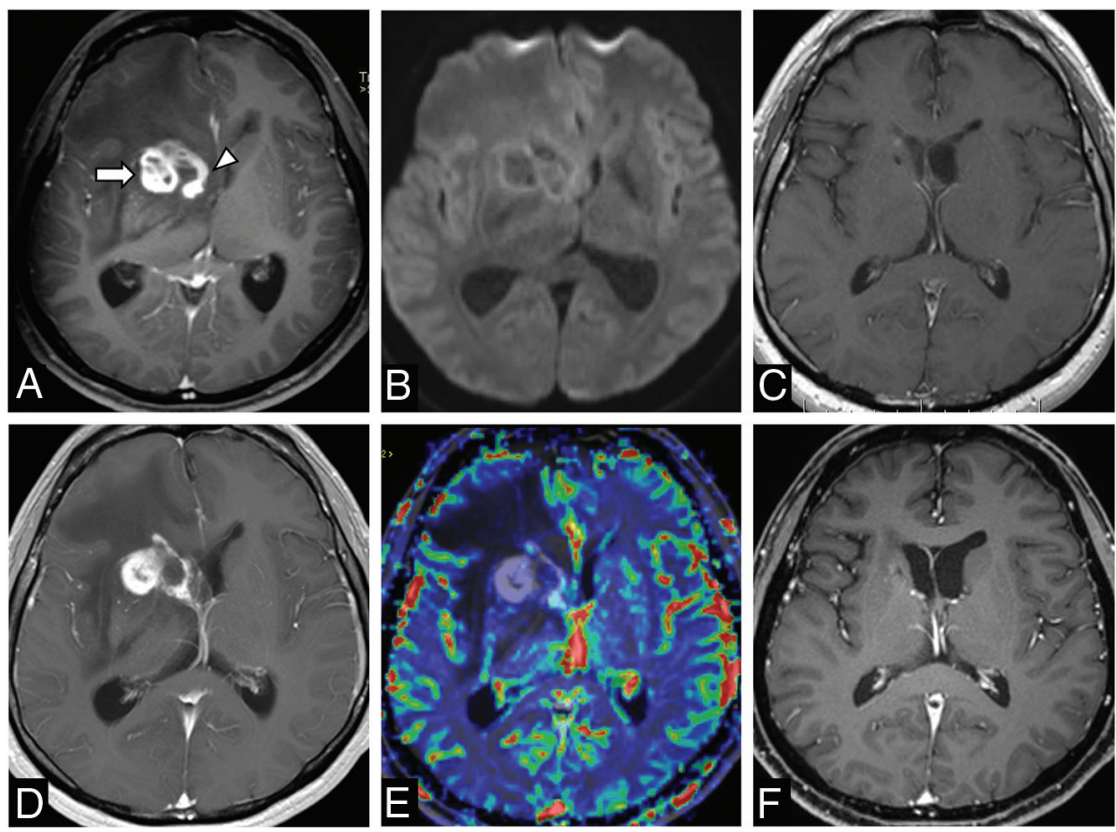

FIG 3. Recurrent granulomatous t-PCNSV. A 30-year-old man presented with headaches (case 4). $A$ and $B$ : Initial brain MR imaging shows conglomerate ring-enhancing mass centered in the right basal ganglia (arrow) with subependymal enhancement (arrowhead) on axial post gadolinium T1weighted images (Gd-TTWI) (A). This mass does not show diffusion restriction on DWI (B). He underwent stereotactic-guided biopsy and histopathology showed granulomatous t-PCNSV. He was treated with corticosteroid and immunosuppressive agents. C: Follow-up MR imaging at 12 months shows the resolution of the disease. $D$ and $E$ : Follow-up MR imaging at 48 months demonstrates recurrent disease in the same region on Gd-TTWI (D). This mass does not show an increase in relative cerebral blood volume on dynamic susceptibility contrast MR perfusion $(E)$. He was again treated with corticosteroids and an immunosuppressive agent. F: Follow-up GdTIWI at 77 months confirms the remission of the disease.

we did not incorporate $\mathrm{A} \beta$-related PCNSV or ABRA/CAA-RI in our analysis because these have recognized characteristic imaging patterns described in prior literature. ${ }^{27-30}$

Our t-PCNSV cohort had a male predominance, which was observed in previous studies. ${ }^{3,6,8,25}$ In our cohort, the most common presentations of patients with t-PCNSV were headaches, seizures, and focal neurologic deficits, which were similar to prior studies. ${ }^{6-8,25}$

In our study, even though-PCNSV had a variety of imaging patterns, we could identify several imaging features shared by many of the patients with t-PCNSV, which may help raise the possible diagnosis of this disease. We found that in t-PCNSV, a single lesion was more common than multiple lesions. The most common location of t-PCNSV was the supratentorial cortex/subcortical areas, as shown in previous studies. ${ }^{25}$ Deep gray nuclei involvement can be seen occasionally. Only 1 patient had brain stem involvement. All patients with t-PCNSV in our cohort demonstrated enhancement, which had various patterns. Patchy and nodular enhancement patterns were the most common. Less common were ring and linear/ perivascular enhancement, respectively. Some patients who demonstrated patchy enhancement had multifocal small hypoenhancing areas within a background of patchy enhancement, resembling a "mottled appearance" (Fig $1 A-C$ ). We hypothesized that this unique pattern of enhancement may represent superimposed small infarctions or necrosis within the background of brain parenchymal inflammatory processes. A minority of the patients showed subependymal or leptomeningeal enhancement associated with the main lesions, thought to represent inflammatory processes involving adjacent leptomeningeal vessels and subependymal vessels, respectively.

Interestingly, in our study, we found that none of the patients with t-PCNSV demonstrated diffusion restriction on DWI (Fig 3B), which several previous studies have reported, ${ }^{6,8,10}$ despite frequent presence of microinfarctions on histopathology. ${ }^{26}$ We could hypothesize that this may reflect the involvement of very small parenchymal/leptomeningeal vessels (including arterioles, capillaries, and/or venules) found in combination with extensive brain parenchymal inflammation, in contrast to medium or large vessel occlusions causing "bland" acute infarctions (54\%-85\%).,

In our study, we found that most patients with t-PCNSV demonstrated microhemorrhages within the lesions, which can be detected on either GRET2* or SWI (Figs $1 E-H$ ). The microhemorrhages can appear either punctate or linear. The pathophysiology of parenchymal microhemorrhages in $\mathrm{t}$ PCNSV is not well understood; one proposed mechanism is the alteration of vessel wall permeability secondary to the vasculitis process itself. $^{9}$

A minority of our patients with t-PCNSV had available advanced MR imaging. Three patients underwent MRP (Fig 3E). There was a low rCBV value that could be due to a lack of neoangiogenesis unlike malignant brain neoplasms, concurring with previous case reports on MRP of t-PCNSV. ${ }^{14,17,20}$ Two patients underwent MR spectroscopy, which showed a nonspecific pattern and did not add specific findings helpful in the exclusion of neoplasm from the diagnosis. ${ }^{10,13,14,18,21,25}$ Three patients had normal vessel wall MR imaging of the large arteries in the circle of Willis.

Our study showed that most t-PCNSV was lymphocytic vasculitis $(90 \%)$, while only $10 \%$ was granulomatous vasculitis. This corresponds to some previous studies. ${ }^{8,10,25}$ However, other previous studies $^{6,7}$ reported that granulomatous vasculitis was more common in PCNSV with a tumorlike presentation. Those studies ${ }^{6,7}$ included patients with ABRA/CAA-RI, which commonly have granulomatous vasculitis and $\mathrm{A} \beta$ deposits in the vessel wall.

In our study, we did not include the patient who was diagnosed with ABRA/CAA-RI. Some previous studies ${ }^{1,5-7,27,30}$ have classified ABRA/CAA-RI as a specific subgroup of t-PCNSV. Although ABRA/CAA-RI share similar patterns of angiocentric inflammatory cell infiltrates of vessel walls, the pathophysiologic mechanism is different as it involves $\mathrm{A} \beta$ deposition in the walls of small-to-medium vessels mostly arteries in the leptomeninges 
and cerebral cortex. ${ }^{5,27}$ It is not fully understood whether the deposition of $\mathrm{A} \beta$ in vessel walls incites the inflammatory response or, conversely, if the inflammation leads to $\mathrm{A} \beta$ deposition. These entities have characteristic imaging patterns described as lobar subcortical edema and cortical/subcortical microhemorrhages associated with predominant leptomeningeal enhancement. ${ }^{27-30}$ ABRA/CAARI rarely show parenchymal enhancement seen in our patients with t-PCNSV. The pattern of microhemorrhage is also usually more diffuse in ABRA/CAA-RI as compared with our patients with $t$ PCNSV, where it was preferentially confined to the areas of signal abnormality and enhancement. An additional reason to exclude ABRA/CAA-RI cases is that ABRA/CAA-RI were commonly seen in older men and those who have other comorbid illnesses and tend to have poorer outcomes compared with t-PCNSV. Furthermore, previous studies in some patients with Alzheimer disease who received amyloid-modifying therapies were found to have amyloidrelated imaging abnormalities, which resemble ABRA/CAA-RI on MR imaging. ${ }^{31,32}$ Its potential mechanism is that immunotherapies cause an increase of clearance of parenchymal plaque $\mathrm{A} \beta$ with a transient increase in vascular $\mathrm{A} \beta$, leading to temporary vascular leakage. This supports the hypothesis that $\mathrm{A} \beta$ plays an important role in the pathomechanism of ABRA/CAA-RI. ${ }^{31,32}$ Therefore, we postulated that ABRA/CAA-RI, where $\mathrm{A} \beta$ deposits are presumed to be the causative factor, could represent an entity different from tPCNSV, where these deposits are absent.

Differentiation between t-PCNSV and brain neoplasms on imaging alone can be challenging. Advanced imaging techniques such as MRP and MR spectroscopy may aid in the diagnosis. $\mathrm{t}$-PCNSV does not show increased relative CBV, which is a feature of neoangiogenesis seen in high-grade gliomas. Differential diagnosis based on imaging findings would often include primary CNS lymphoma (PCNSL). Intralesional microhemorrhages are not an uncommon imaging feature of PCNSL. ${ }^{33}$ Furthermore, a previous study has shown that systemic lymphoma can occur in about $6 \%$ of patients with PCNSV. ${ }^{34}$ These patients may have a prior history of systemic lymphoma or simultaneous systemic lymphoma detected on PCNSV work-up. ${ }^{34}$ Radiologically, the lack of diffusion restriction may be helpful in differentiating tPCNSV from PCNSL. In our cohort, none of our patients had systemic lymphoma, either at the time of the biopsy or on followup. The presence of intralesional microhemorrhages and lack of specific open ring enhancement or peripheral diffusion restriction assisted in differentiating t-PCNSV from tumefactive demyelinating lesions. ${ }^{35}$ Other unusual atypical infectious conditions such as CNS tuberculosis and fungal infections should also be included in the differential diagnosis of t-PCNSV.

Our study has several limitations; first, as a retrospective study the imaging protocols applied were heterogeneous and some patients did not have complete laboratory tests and follow-up. Second, the extreme rarity of t-PCNSV prevented the undertaking of large-scale studies. We collected cases from different institutions, which may have caused a selection bias. Third, PCNSV has a different disease subtype and multifaceted clinical and histopathologic appearances resulting in difficulty in the grouping of imaging appearances for the diagnosis. Furthermore, we did not have available DSA, which is considered the reference standard for the preoperative diagnosis of PCNSV, because those patients were thought to have tumors preoperatively. Once the histopathologic diagnosis of PCNSV has been established, DSA is usually not deemed necessary especially in the era of CTA and MRA. It would have been helpful if there were available DSA, though DSA is often negative if the affected vessel diameter is less than $200 \mu \mathrm{m}$ in diameter. ${ }^{36}$ In addition, previous studies have already shown that most patients in this subgroup have normal DSA findings. $4,6-8$

\section{CONCLUSIONS}

T-PCNSV is a distinct subgroup of small vessel vasculitides that can closely mimic brain neoplasm. Pertinent differentiating imaging features include a cortical/subcortical enhancing masslike lesion with intralesional punctate/linear microhemorrhages, lack of diffusion restriction, and low rCBV. Importantly, absent largemedium vessel involvement on CTA/MRA should not exclude $\mathrm{t}$-PCNSV. Open biopsy and histopathologic examination remain the "gold standard" for definitive diagnosis.

Disclosures: Suradech Suthiphosuwan-UNRELATED: Grants/Grants Pending: Sanofi-Genzyme, Comments: Fellowship educational support by unrestricted educational grant.* Anne Osborn-UNRELATED: Payment for Lectures Including Service on Speakers Bureaus: Bracco Visiting Professor, Stanford University, Comments: Lectures, case conferences March 1-2, 2020; Royalties: Reed-Elsevier, Comments: Author, multiple books and STATdx contributions. *Money paid to the institution.

\section{REFERENCES}

1. Hajj-Ali RA, Singhal AB, Benseler $S$, et al. Primary angiitis of the CNS. Lancet Neurol 2011;10:561-72 CrossRef Medline

2. Twilt M, Benseler SM. The spectrum of CNS vasculitis in children and adults. Nat Rev Rheumatol 2011;8:97-107 CrossRef Medline

3. Becker J, Horn PA, Keyvani K, et al. Primary central nervous system vasculitis and its mimicking diseases - clinical features, outcome, comorbidities and diagnostic results - a case control study. Clin Neurol Neurosurg 2017;156:48-54 CrossRef Medline

4. Thaler C, Kaufmann-Buhler AK, Gansukh T, et al. Neuroradiologic characteristics of primary angiitis of the central nervous system according to the affected vessel size. Clin Neuroradiol 2019;29:37-44 CrossRef Medline

5. Salvarani C, Brown RD, Jr., Christianson T, et al. An update of the Mayo Clinic cohort of patients with adult primary central nervous system vasculitis: description of 163 patients. Medicine (Baltimore) 2015;94:e738 CrossRef Medline

6. Salvarani C, Brown RD, Jr., Christianson TJH, et al. Primary central nervous system vasculitis mimicking brain tumor: comprehensive analysis of 13 cases from a single institutional cohort of 191 cases. J Autoimmun 2019;97:22-28 CrossRef Medline

7. Molloy ES, Singhal AB, Calabrese LH. Tumour-like mass lesion: an under-recognised presentation of primary angiitis of the central nervous system. Ann Rheum Dis 2008;67:1732-35 CrossRef Medline

8. de Boysson H, Boulouis G, Dequatre N, et al. Tumor-like presentation of primary angiitis of the central nervous system. Stroke 2016;47:2401-04 CrossRef Medline

9. Boulouis G, de Boysson H, Zuber M, French Vasculitis Group, et al. Primary angiitis of the central nervous system: magnetic resonance imaging spectrum of parenchymal, meningeal, and vascular lesions at baseline. Stroke 2017;48:1248-55 CrossRef Medline

10. Lee $\mathrm{Y}, \mathrm{Kim} \mathrm{JH}, \mathrm{Kim} \mathrm{E}$, et al. Tumor-mimicking primary angiitis of the central nervous system: initial and follow-up MR features. Neuroradiology 2009;51:651-59 CrossRef Medline

11. Denny AM, Das SK. A case of central nervous system vasculitis presenting as a mass-like lesion. Childs Nerv Syst 2019;35:1223-26 CrossRef Medline 
12. Killeen T, Jucker D, Went $\mathrm{P}$, et al. Solitary tumour-like mass lesions of the central nervous system: primary angiitis of the CNS and inflammatory pseudotumour. Clin Neurol Neurosurg 2015;135:3437 CrossRef Medline

13. Kumar PP, Rajesh A, Kandadai RM, et al. Primary CNS vasculitis masquerading as glioblastoma: a case report and review. Asian J Neurosurg 2017;12:69-71 CrossRef Medline

14. Lee JS, Jung TY, Lee KH, et al. Primary central nervous system vasculitis mimicking a cortical brain tumor: a case report. Brain Tumor Res Treat 2017;5:30-33 CrossRef Medline

15. Lyra TG, Martin MGM, Carvalho RC, et al. Pseudotumoral presentation of primary central nervous system vasculitis. Arq Neuropsiquiatr 2013;71:333-35 CrossRef Medline

16. Mabray MC, Cha S. CNS angiitis as a brain tumor mimic with a branching vascular abnormality on T2* MRI. Neurology 2015;85:181920 CrossRef Medline

17. Muccio CF, Di Blasi A, Esposito G, et al. Perfusion and spectroscopy magnetic resonance imaging in a case of lymphocytic vasculitis mimicking brain tumor. Pol J Radiology 2013;78:66-69 CrossRef Medline

18. Panchal NJ, Niku S, Imbesi SG. Lymphocytic vasculitis mimicking aggressive multifocal cerebral neoplasm: MR imaging and MR spectroscopic appearance. AJNR Am J Neuroradiol 2005;26:642-45 Medline

19. Sun LI, Zhu L, Zhao T, et al. A rare case of tumor-mimicking primary angiitis of the central nervous system. Mol Clin Oncol 2016;4:827-29 CrossRef Medline

20. Tanei T, Nakahara N, Takebayashi S, et al. Primary angiitis of the central nervous system mimicking tumor-like lesion-case report. Neurol Med Chir (Tokyo) 2011;51:56-59 CrossRef Medline

21. Zhang G, Yang C, Chang J, et al. Primary angiitis of the central nervous system mimicking a cerebellar tumor. Br J Neurosurg 20181-83 CrossRef Medline

22. Zhu DS, Yang XL, Lv HH, et al. Seizure syndrome as a first manifestation of solitary tumor-like mass lesion of PACNS: two case reports. Medicine (Baltimore) 2017;96:e6018 CrossRef Medline

23. Qu SB, Khan S, Liu H. Primary central nervous system vasculitis mimicking brain tumour: case report and literature review. Rheumatol Int 2009;30:127-34 CrossRef Medline

24. You G, Yan W, Zhang W, et al. Isolated angiitis of the central nervous system with tumor-like lesion, mimicking brain malignant glioma: a case report and review of the literature. World J Surg Oncol 2011;9:97 CrossRef Medline
25. Jin $\mathrm{H}, \mathrm{Qu} \mathrm{Y}, \mathrm{Guo} \mathrm{ZN}$, et al. Primary angiitis of the central nervous system mimicking glioblastoma: a case report and literature review. Front Neurol 2019;10:1208 CrossRef Medline

26. Giannini C, Salvarani C, Hunder G, et al. Primary central nervous system vasculitis: pathology and mechanisms. Acta Neuropathol 2012;123:759-72 CrossRef Medline

27. Salvarani C, Hunder GG, Morris JM, et al. A $\beta$-related angiitis: comparison with CAA without inflammation and primary $\mathrm{CNS}$ vasculitis. Neurology 2013;81:1596-1603 CrossRef Medline

28. Salvarani C, Morris JM, Giannini C, et al. Imaging findings of cerebral amyloid angiopathy, abeta-related angiitis (ABRA), and cerebral amyloid angiopathy-related inflammation: a single-institution 25year experience. Medicine (Baltimore) 2016;95:e3613 CrossRef Medline

29. Miller-Thomas MM, Sipe AL, Benzinger TL, et al. Multimodality review of amyloid-related diseases of the central nervous system. Radiographics 2016;36:1147-63 CrossRef Medline

30. Moussaddy A, Levy A, Strbian D, et al. Inflammatory cerebral amyloid angiopathy, amyloid-beta-related angiitis, and primary angiitis of the central nervous system: similarities and differences. Stroke 2015;46:e210-13 CrossRef Medline

31. Sperling RA, Jack CR, Jr., Black SE, et al. Amyloid-related imaging abnormalities in amyloid-modifying therapeutic trials: recommendations from the Alzheimer's Association Research Roundtable Workgroup. Alzheimers Dement 2011;7:367-85 CrossRef Medline

32. Barakos J, Sperling R, Salloway S, et al. MR imaging features of amyloid-related imaging abnormalities. AJNR Am J Neuroradiol 2013;34:1958-65 CrossRef Medline

33. Sakata A, Okada T, Yamamoto A, et al. Primary central nervous system lymphoma: is absence of intratumoral hemorrhage a characteristic finding on MRI? Radiology and Oncology 2015;49:128-34 CrossRef Medline

34. Salvarani C, Brown RD, Jr, Christianson TJH, et al. Primary central nervous system vasculitis associated with lymphoma. Neurology 2018;90:e847-55 CrossRef Medline

35. Suh $\mathrm{CH}$, Kim HS, Jung SC, et al. MRI findings in tumefactive demyelinating lesions: a systematic review and meta-analysis. AJNR Am J Neuroradiol 2018;39:1643-49 CrossRef Medline

36. Brant-Zawadzki M, Gould R, Norman D, et al. Digital subtraction cerebral angiography by intraarterial injection: comparison with conventional angiography. AJNR Am J Neuroradiol 1982;3:593-99 Medline 